\title{
THE SIMPLE-ZERO CONJECTURE FOR SUPPORT POINTS IN $\Sigma$
}

\section{Y. J. LEUNG AND G. SCHOBER}

Denote by $\Sigma$ the class of functions $f(z)=z+\sum_{n=0}^{\infty} b_{n} z^{-n}$ analytic and univalent in $\Delta=\{z:|z|>1\}$. Let $L$ be a continuous linear functional defined on the space of functions analytic in $\Delta$, with the topology of locally uniform convergence. Linear combinations of coefficients and, more generally, linear combinations of point evaluations of the function and its derivatives are typical examples of continuous linear functionals. A function $f$ in $\Sigma$ is called a support point of $\Sigma$ for $L$ if $\operatorname{Re} L(f) \geq \operatorname{Re} L(g)$ for all $g$ in $\Sigma$ and $\operatorname{Re} L(f)>\operatorname{Re} L(g)$ for some $g$ in $\Sigma$. The simple-zero conjecture concerns the geometric structure of the continuum $\Gamma=\mathbf{C} \backslash f(\Delta)$ omitted by a support point $f$.

The mappings of the class $\Sigma$ arise, for example, in flow problems past an obstacle. In this sense, the class $\Sigma$ is more natural than the familiar schlicht class $S$ of normalized univalent functions in $|z|<1$. While the de Branges theorem asserts that the single function $k(z)=\sum_{n=1}^{\infty} n z^{n}$ solves the coefficient problem for $S$, the situation for $\Sigma$ appears to be more difficult. In particular, a sharp bound for $b_{n}$ is known only for $1 \leq n \leq 3$. One obstacle seems to be that the extremal functions change with $n$. Another is their nonelementary nature. In fact, there are relatively few conjectures (cf. [10]).

Therefore, it is natural to look first for qualitative information. A number of properties are known, even about support points of $\Sigma$. For example, it follows from Schiffer's boundary variation (cf. [9]) that the set $\Gamma$ omitted by a support point $f$ corresponding to a linear functional $L$ consists of analytic arcs lying on trajectories of the quadratic differential $L(1 /(f-w)) d w^{2}$. Trajectories of $Q(w) d w^{2}$ are $\operatorname{arcs} w=w(t)$ on which $Q(w(t)) w^{\prime}(t)^{2}>0$, together with their endpoints. As a function of $w, Q(w)=L(1 /(f-w))$ has an analytic extension to some open region containing the omitted set $\Gamma$. Thus the structure theory of quadratic differentials (cf. [11]) shows that the arcs of $\Gamma$ are joined to one another only at the zeros of $L(1 /(f-w))$. At a zero of order $k \geq 1$, at most $k+2$ arcs may join at a time in a subset of $k+2$ equiangular directions. For example, the coefficient functional $L(f)=b_{3}$ leads to a quadratic differential of the form $\left(w^{2}-b_{1}\right) d w^{2}$. The omitted set of a support point for this functional forks at each of the two simple zeros at $\pm \sqrt{b_{1}}$ in three equi-angular directions [4]. Further examples of support points can be found in $[\mathbf{1}, \mathbf{5}, \mathbf{6}, \mathbf{1 0}]$. Based on these examples, one might conjecture that the quadratic differential $L(1 /(f-w)) d w^{2}$ associated with a support point $f$ in $\Sigma$ can have only simple zeros on the set $\Gamma$ omitted by $f$. It is the purpose

Received by the editors April 13, 1988.

1980 Mathematics Subject Classification (1985 Revision). Primary 30C75.

This work was supported in part by grants from the National Science Foundation. 
of this note to announce that this remarkable property is true for the most general support point of $\Sigma$.

THEOREM. If $f$ is a support point of $\Sigma$ for some linear functional $L$, then its associated quadratic differential $L(1 /(f-w)) d w^{2}$ can have only simple zeros on the set $\Gamma$ omitted by $f$. Thus, at the points where $\Gamma$ forks, it does so in at most three equi-angular directions.

The proof [8] of the Theorem consists of two parts. First, we use a technique of Schiffer's $[\mathbf{3}, \mathbf{7}]$ to convert the conjecture into a coefficient problem. Very briefly, if the omitted set of a support point for some functional contains a $k$ th order zero of its associated quadratic differential, then a variational argument leads to the inequality $\operatorname{Re}\left\{C_{k+1}\right\} \leq 0$ for every function $F$ analytic and univalent in the complement of the line segment $[0,4]$ with expansion $F(w)=w+\sum_{j=0}^{\infty} C_{j} w^{-j}$ in a neighborhood of infinity. Next, we show that there are functions which violate this inequality for $k \geq 2$ by a technique which is in the spirit of E. Bombieri's [2] second variation of the Koebe function in terms of Loewner's differential equation.

\section{REFERENCES}

1. Y. Abu-Muhanna and Y. J. Leung, On analytic slit mappings in the class $\Sigma$, Proc. Amer. Math. Soc. 99 (1987), 44-48.

2. E. Bombieri, On the local maximum property of the Koebe function, Invent. Math. 4 (1967), 26-67.

3. A. Chang, M. M. Schiffer, and G. Schober, On the second variation for univalent functions, J. Analyse Math. 40 (1981), 203-238.

4. P. R. Garabedian and M. Schiffer, A coefficient inequality for schlicht functions, Ann. of Math. (2) 61 (1955), 116-136.

5. W. E. Kirwan and G. Schober, New inequalities from old ones, Math. Z. 180 (1982), $19-40$.

6. Y. J. Leung and G. Schober, Low order coefficient estimates in the class $\Sigma$, Ann. Acad. Sci. Fenn. Ser. AI Math. 11 (1986), 36-61.

7. _, On the structure of support points in the class $\Sigma$, J. Analyse Math. 46 (1986), 176-193.

8. — The simple-zero theorem for support points in $\Sigma$, Proc. Amer. Math. Soc. (to appear).

9. G. Schober, Univalent functions-Selected topics, Lecture Notes in Math., vol. 478, Springer-Verlag, Berlin and New York, 1975.

10. __ Some conjectures for the class $\Sigma$, Topics in Complex Analysis, Contemp. Math., vol. 38, Amer. Math. Soc., Providence, R. I., 1985, pp. 13-21.

11. K. Strebel, Quadratic differentials, Springer-Verlag, Berlin and New York, 1984.

Department of Mathematics, University of Delaware, NeWARK, DelaWARE 19716

DEPARTMENT OF MATHEMATICS, INDIANA UNIVERSity, BloOMINGTON, INDIANA 47405 